\title{
Louanne Hudgins, Helga V. Toriello, Gregory M. Enns and H. Eugene Hoyme (eds): Signs and Symptoms of Genetic Conditions, a Handbook
}

\author{
Oxford University Press 2014, 560 pages, 978-0-19-993097-5, Paperback
}

\author{
Ian Tully ${ }^{1}$
}

Published online: 26 July 2015

(C) Springer-Verlag Berlin Heidelberg 2015

In this, the first edition of the handbook, the editors bring their combined acumen in training and education to bear on the clinical practice of genetics. From the introduction onwards, this book sets out its stall as a companion for the clinical approach to the genetics patient, rather than an exhaustive scientific reference.

Given the vast number of rare genetic conditions available to write about, the task of describing the whole field in a concise text is impossible. Instead, this text offers an overview of genetics, grouped into separate chapters by symptomatology.

The chapters are well written, concise and informative. The tables are well laid out, and the algorithmic charts are of particular use to anybody looking to develop a more systematic approach to the presentation of genetic disorders.

A few chapters lean heavily on long tabulated lists, which may be daunting to a clinician with minimal experience in the field. Given the importance of 'Gestalt' recognition in genetic diagnosis, some of the space spent on tabulation could have been better used for high-quality clinical photographs. These, however, are minor criticisms of an otherwise excellent clinical text.

As may be fitting for a book focussing on signs and symptoms, minimal emphasis is put on the subject of whole exome and whole genome sequencing (WES and WGS) technologies. The editors justify this decision at length in their introduction. However as these tests move to the first line of investigations, the handbook will require extensive review. A significant benefit of this approach is that it highlights the need for engagement with the limitations of this technology and the avoidance of its implementation as a catch-all diagnostic tool that avoids the need for thought and clinical experience.

The book is also relatively limited in its exploration of current first-line testing, with array $\mathrm{CGH}$ given the same level of explanation as the amino acid profile. Drawing attention to the challenges posed by interpretation of genetic tests, particularly the dreaded variant of uncertain significance, would provide a deeper understanding of the process of genetic diagnosis.

As a vade mecum for professionals looking to develop their approach to clinical genetics, this book will prove invaluable. It will be of particular use to junior trainees in the practice of medical genetics, as well as advanced trainees in paediatric and adult medicine. Limited focus on the investigations is a drawback, but as a guide to the "signs and symptoms" of genetic conditions, this is a first rate tool.
Ian Tully

Ian.Tully2@wales.nhs.uk

1 All Wales Medical Genetic Service, Institute of Medical Genetics, University Hospital of Wales, Cardiff, UK 